\title{
Recent results and status of the XENON program
}

\section{J. Masbou* on behalf of the XENON Collaboration}

Subatech, Ecole des Mines de Nantes, CNRS/In2p3, Université de Nantes, Nantes, France

E-mail: julien.masbou@subatech.in2p3.fr

The XENON program aims at the direct detection of dark matter WIMPs with liquid xenon as target and detecting material. With detectors of increasing target mass and decreasing background, XENON has achieved competitive limits on WIMP-nucleon interaction couplings, but also on axions and axion-like particles. The detector has been ongoing at the Laboratori Nazionali del Gran Sasso in Italy since 2009 with a dual phase (liquid-gas) xenon Time Projection Chamber and a total mass of $161 \mathrm{~kg}$ of liquid xenon. The most recent results will be presented. Current run mainly focuses on additional calibration for the low energy response of the detector and the validation of new calibration techniques in view of the next generation experiment, XENON1T. XENON1T will be the first experiment to use liquid xenon in a time projection chamber at the ton scale. It is designed to achieve two orders of magnitude higher sensitivity than the current best limits.

The 34th International Cosmic Ray Conference,

30 July- 6 August, 2015

The Hague, The Netherlands

\footnotetext{
* Speaker.
} 


\section{Introduction}

The existence of a non-luminous, non-baryonic and cold (i.e. non-relativistic) matter is suggested by an increasing number of astrophysical and astronomical observations. This Cold Dark Matter (CDM) [1][2] represent about 26\% of the whole energy budget of the universe. One of the most plausible candidate for CDM are Weakly Interactive Massive Particles (WIMPs). They are predicted by extention of the standard of electroweak interaction: supersymmetric theories (SUSY), extra dimensions and little Higgs models [3][4]

In order to determine the characteristics of such particles, three main approaches are developped. Particle accelerators, like LHC, try to create them in the collisions. High energy astroparticle physics experiments look for WIMP annihilation signatures using mainly gamma-rays, neutrinos and anti-matter. Dark matter direct detection experiments want to observe the elastic scattering of WIMPS on the detector's nuclei.

XENON10 and XENON100 [5] where the first successful results with 10 and $100 \mathrm{~kg}$ scale liquid xenon detectors. The new project with multi-ton liquid xenon, XENON1T, will continue to lead this field. It expected sensitivity to the spin-independent WIMP-nucleon cross section of $2 \times 10^{-47} \mathrm{~cm}^{2}$. XENON1T is currently in advanced phase of construction at Laboratori Nazionali del Gran Sasso in Italy (LNGS).

\section{XENON100}

Several reasons motivated the use of liquid xenon (LXe) as target for a dark matter direct search experiment: it has a high density $\left(\rho \sim 3 \mathrm{~g} / \mathrm{cm}^{3}\right.$ ), easily allowing for scalability to ton scale detectors of relatively small sizes; it high atomic number $(\mathrm{A} \sim 131)$ which enhances the probability of WIMP-nucleus spin-independent interactions $\left(\sigma \propto \mathrm{A}^{2}\right)$. Odd isotopes are naturally present in xenon with $\sim 50 \%$ abundance. It allows to probe also the WIMP-nucleon spin dependent interactions. A low energy threshold is achievable thanks to the high ionization and scintillation yield in liquid xenon. Moreover the requirement of low background is reachable thanks to the absence of any long lived radioactive isotope in the noble liquid and to its efficiency as self-shielding target, given the high $\mathrm{Z}$ (54). This feature, combined with the possibility of reconstructing the position of the interactions in $3 \mathrm{D}$, allows background reduction by volume fiducialization.

The XENON100 detector is a dual-phase (liquid-gas) Time Projection Chamber (TPC) filled with xenon: $\sim 62 \mathrm{~kg}$ of active volume surrounded by $\sim 99 \mathrm{~kg}$ of liquid xenon acting as a veto [6]. The TPC is enclosed in a PTFE cylinder of $30 \mathrm{~cm}$ height and $\sim 30 \mathrm{~cm}$ diameter (PTFE is a suitable fluoropolymer with very good UV light reflective features [7]). The active volume is viewed by a total of 242 squared (1" $\times 1$ ") low radioactivity photo-multiplier tubes (Hamamatsu R8520-06-Al), especially developed to be operational at the liquid xenon temperature for the characteristic xenon scintillation wavelength $(\lambda=178 \mathrm{~nm}) .98$ of these PMTs are on the top of the TPC in the gas phase, arranged in concentric circles to improve position reconstruction, whereas 80 PMTs are on the bottom of the TPC placed very close to each other in order to optimize the light collection. The remaining 64 PMTs are placed in the veto region, looking at the side, at the top and at bottom around the TPC. 
The detector is located underground at the LNGS in Italy, under $1400 \mathrm{~m}$ (3600 meters water equivalent) of rock, where the muon flux is reduced by six orders of magnitude compared to the surface. All the detector construction materials were selected for low radioactivity [8] and cryogenic components and electrical feedthroughs were placed outside of a low radioactivity shield, made of $20 \mathrm{~cm}$ of water, $20 \mathrm{~cm}$ of lead, $20 \mathrm{~cm}$ of high density polyethylene and $5 \mathrm{~cm}$ of innermost copper.

The basic working principles of XENON100 are the following (see [6] for more details): a particle interacting in the liquid xenon target generates a prompt scintillation signal (S1) which is readily detected by the two arrays of PMTs. The ionization electrons produced in the same interaction are drifted towards the anode, in an electric field across the TPC $(\sim 530 \mathrm{~V} / \mathrm{cm})$. Once they reach the liquid-gas interface, given the high $(\sim 12 \mathrm{kV} / \mathrm{cm})$ field in the $\sim 2.5 \mathrm{~mm}$ gas gap, such electrons are extracted from the liquid and accelerated in the gas, producing a secondary scintillation signal (S2), which is proportional to the ionization charge, thus also detected by the two arrays of PMTs. The time distance between S1 and S2 (drift time), since the electron drift velocity in liquid xenon is known $(\sim 1.74 \mathrm{~mm} / \mu \mathrm{s}$ at $0.53 \mathrm{kV} / \mathrm{cm})$, gives the information of the position of the interaction vertex in the vertical axis. Since the diffusion of electrons in the liquid is very small and the S2 signal is very localized on the top, the hit pattern of the secondary scintillation signal in the top PMT array can be used in order to reconstruct the interaction vertex in the horizontal plane. Three different methods are used to reconstruct the XY position, which are based on $\chi^{2}$ minimization, neural network and support vector machine algorithms. In conclusion a 3-dimensional event position information is available. The nature of the impinging particles can be discriminated by studying the S1 and S2 signals. For electron recoils (expected background in the energy region of interest for dark matter search) the ratio of S2/S1 is much higher than for nuclear recoils (expected WIMP signal), because of the different ionization density of the two particles. This feature is used to discriminate between electronic recoil (ER) background events from nuclear recoil (NR) possibly signal events.

\subsection{Analysis}

Collected between March 2011 and April 2012, the dataset of XENON100 represent 224.6 live days with $34 \mathrm{~kg}$ fiducial mass and include some improvements : a longer exposure, a significantly lower intrinsic ${ }^{85} \mathrm{Kr}$ contamination, an improved electronic noise con- dition and a lower trigger threshold. Regularl calibrations were done with various radioactive sources. ${ }^{232} \mathrm{Th}$ and ${ }^{60} \mathrm{Co}$ sources was used to determine the electronic recoil (ER) background. The nuclear recoil (NR) response of the detector was determined from a neutron source $\left({ }^{241} \mathrm{AmBe}\right)$. The electron lifetime in liquid xenon was estimate from a ${ }^{137} \mathrm{Cs}$ source.

A profile likellihood (PL) approach is used to test the background-only and signal hypothesis. The systematic uncertainties in the energy scale and in the background expectation was profiled out and represented in the limit. Poisson fluctuations in the number of photoelectrons (PEs) dominate the $\mathrm{S} 1$ energy resolution and was also taken into account along with the single PE energy resolution of the PMTs. 


\subsection{WIMPs results}

The signal model is derived from an isothermal halo with a local density of $0.3 \mathrm{GeV} / \mathrm{cm}^{3}$, a local circular velocity of $220 \mathrm{~km} / \mathrm{s}$, and a galactic escape velocity of $544 \mathrm{~km} / \mathrm{s}$. WIMPs interactions can be described in terms of scalar (spin-independent, SI) and axial-vector (spin-dependent, SD) couplings. If the WIMP is a spin-1/2 or a spin-1 field, the contributions to the WIMP-nucleus scattering arise from couplings of the WIMP field to the quark axial current and will couple to the total angular momentum of a nucleus and only nuclei with an odd number of protons or/and neutrons will yield a significant sensitivity to this channel. Two nonzero spin isotopes ${ }^{129} \mathrm{Xe}$ (spin-1/2) and ${ }^{131} \mathrm{Xe}$ (spin-3/2), with an abundance of $26.4 \%$ and $21.2 \%$ respectively are contains in natural xenon. This led to the $90 \%$ C.L. exclusion on spin-independent WIMP-nucleon cross section as can be seen in Fig. 1. The constraints on the SD WIMP-nucleon cross section were derived as shown in Fig. 2. XENON100 was able to exclude WIMP-neutron cross section down to $3.5 \times 10^{-40} \mathrm{~cm}^{2}$ for a WIMP mass of $45 \mathrm{GeV} / \mathrm{c}^{2}$ setting the most stringent limit to date on the WIMP-neutron SD couplings for WIMP masses above $6 \mathrm{GeV} / \mathrm{c}^{2}$.

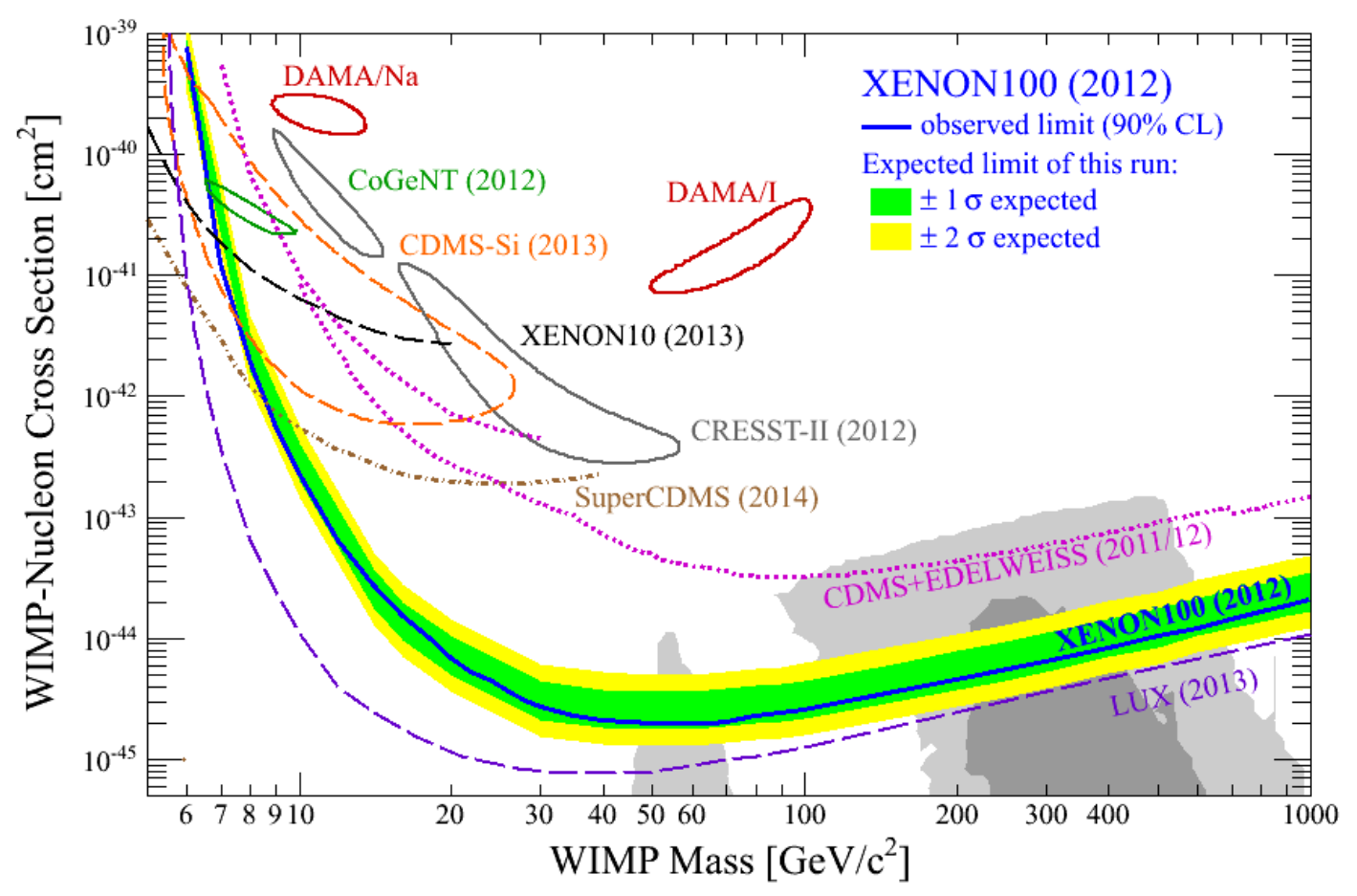

Figure 1: The XENON100 limits (90\% CL) on Spin Independent WIMP-nucleon cross section

\subsection{Solar Axions and Axions Like Particles}

Axions were introduced by Peccei and Quinn to solve the strong CP problem as pseudoNambu-Goldstone bosons emerging from the breaking of a global U(1) symmetry [9]. Although this original model has been ruled out, "invisible" axions arising from a higher symmetry-breaking energy scale are still allowed, as described, for example, in the DFSZ and KSVZ models [10]. In addition to QCD axions, axion-like particles (ALPs) are pseudoscalars that do not necessarily 

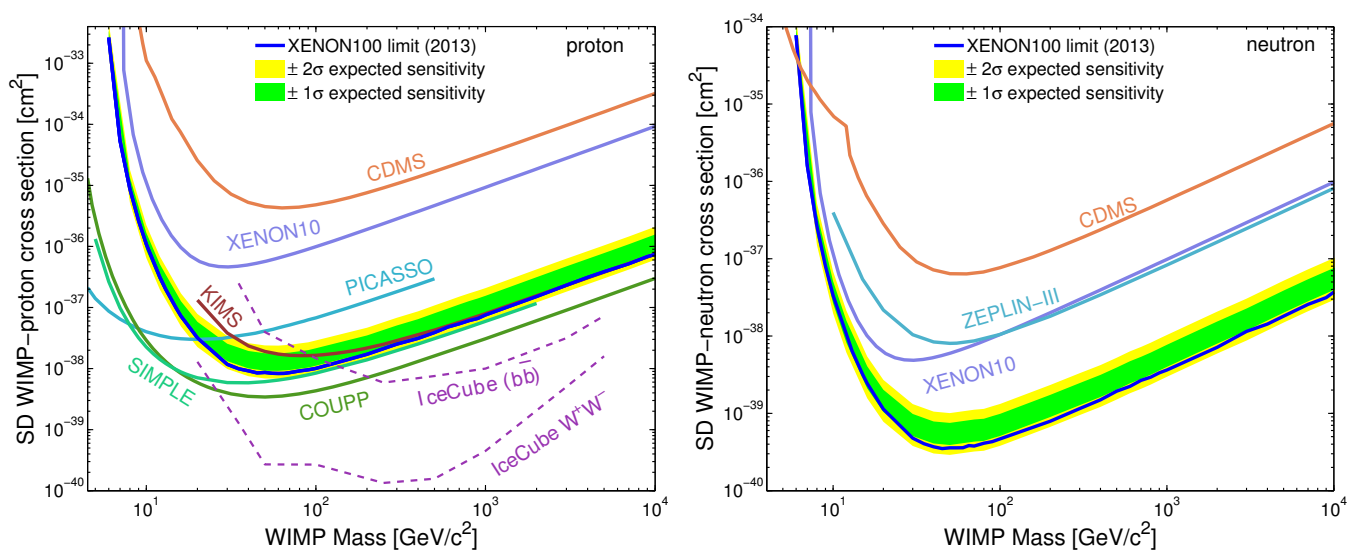

Figure 2: The XENON100 limits (90\% CL) on Spin Dependent WIMP-nucleon cross section

solve the strong $\mathrm{CP}$ problem, but that were introduced by many extensions of the Standard Model of particle physics. Axions as well as ALPs are another class of well motivated cold dark matter candidates [11]. Axions and ALPs may give rise to observable signatures in detectors through their coupling to photons, electrons and nuclei. The coupling to electrons $\mathrm{g}_{A_{e}}$ may be tested via scattering off the electrons of a target, such as liquid xenon (LXe), through the axio-electric effect. This process is the analogue of the photo-electric effect with the absorption of an axion instead of a photon. The first axion search performed with the XENON100 experiment is reported in [12]. Fig. 3 shows the new XENON100 exclusion limit on $\mathrm{g}_{A}$ at $90 \% \mathrm{CL}$ for both solar axions and ALPs.
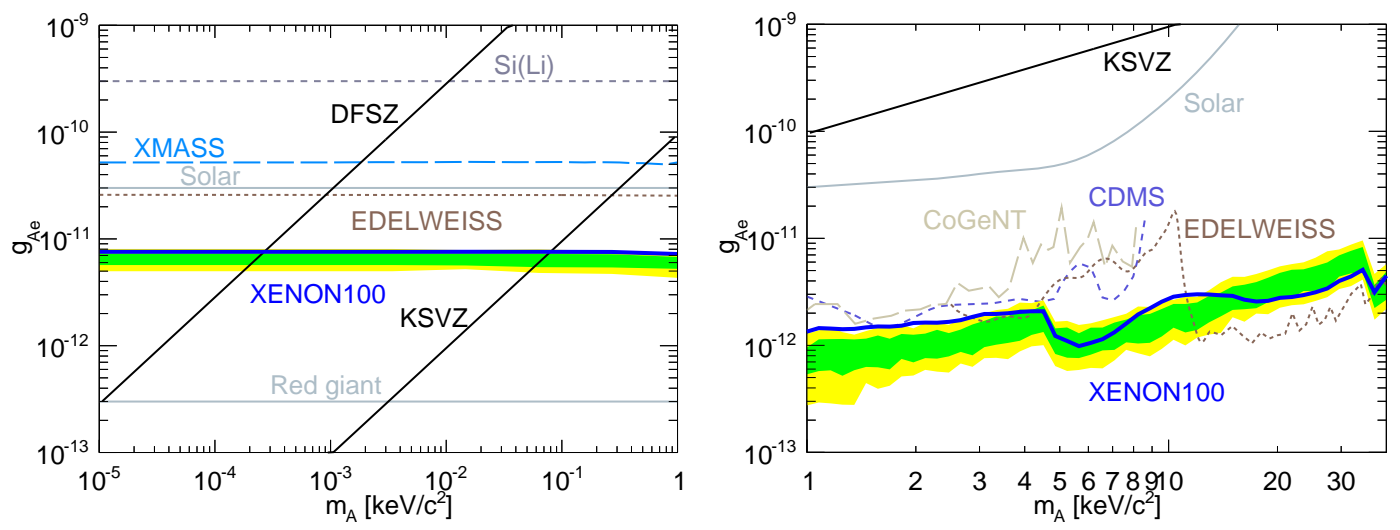

Figure 3: The XENON100 limits $(90 \% \mathrm{CL})$ on the electron coupling constant $\mathrm{g}_{A_{e}}$ for solar axions (left) and on ALPs (right)

\section{XENON1T}

The construction of XENON1T started in 2013 at the LNGS in Italy and it is currently under commissioning phase. Figure 4 (left) shows a picture of the XENON1T facility. Like its predecessors XENON10 and XENON100, the core of the detector is a dual-phase xenon TPC. The 
XENON1T detector will contain $\sim 3.3$ tons of high-purity liquid xenon ( 2.0 tons of which as active volume inside TPC), which provides excellent discrimination of electronic and nuclear interaction. With the self-shielding property of liquid xenon, the outer volume of xenon effectively shields the inner volume from background interactions occurring outside the TPC. This last one will be instrumented from both above and below with a total of 248 low-background photomultiplier tubes (PMT), and its volume will be kept under a homogeneous electric field $E_{d}$ up to $1 \mathrm{kV} / \mathrm{cm}$ over a drift distance of $1 \mathrm{~m}$.
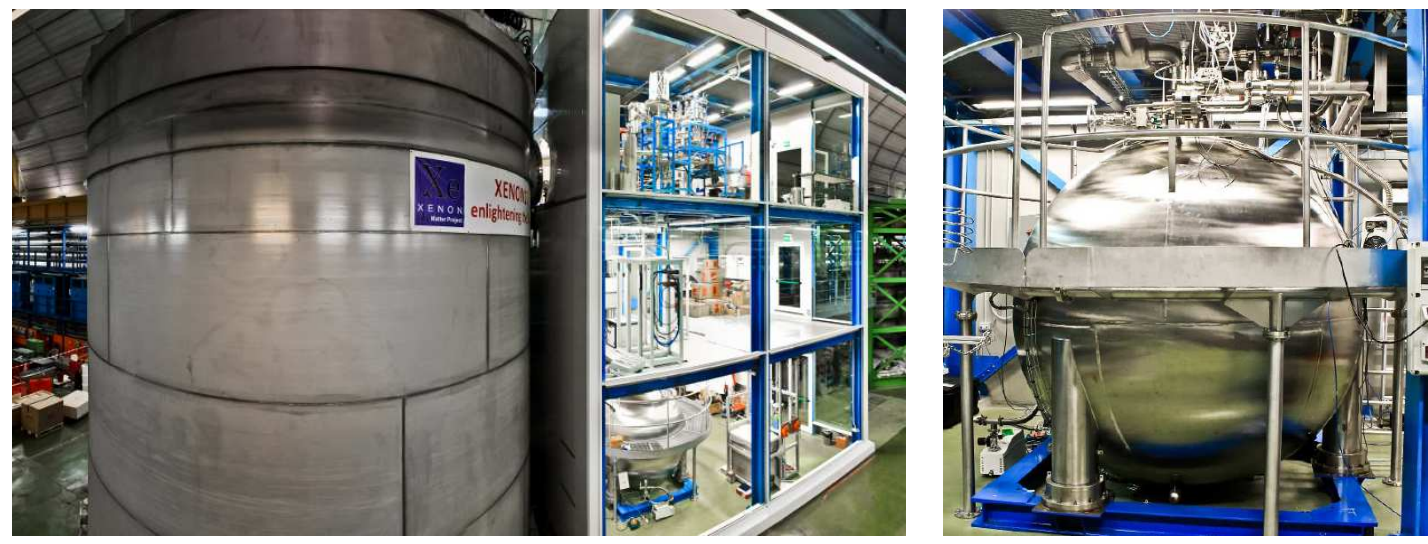

Figure 4: (left) XENON1T installed in Hall B in LNGS, Italy. (right) The XENON1T storage and recovery system (ReStoX).

\subsection{Expected background and sensitivity}

In order to achieve the best sensitivity, XENON1T, designed as a low-rate counting experiment, has to reduce the sources of background to approximately zero level. Cosmic muons are one of these. It produces secondary neutrons as they interact near the detector, thus producing nuclear recoil background. Even if the muon flux is highly reduced at the site (LNGS stays under 3600 m.w.e.), the residual rate of muon-induced neutrons can still affect the sensitivity in the signal region. The TPC and its cryostat will be immersed in $700 \mathrm{t}$ of pure water contained in a stainless steel tank to avoid this. The tank is equipped with an active muon veto with 84 PMTs, allowing a $99 \%$ efficiency in tagging crossing muons and $74 \%$ efficiency for showering events. The total background is expected to be around 0.01 event per year [13]. Figure 4 (left) shows the water tank completed in fall 2013; on the same picture, the XENON1T service building completed at the beginning of 2014 is shown. In order to reduce background contamination it is essential to use radiopure raw material to build the different detector components and for this purpose several screening campaigns were performed and others are ongoing. The choices of the stainless steel used to build the cryostat and of the other materials that will be used for the TPC are indeed outcomes of these screening campaigns. The two arrays of low-background PMTs, 127 on top and 121 on bottom of the TPC, are 3-inches R11410-21 manufactured by Hamamatsu Photonics K.K., characterized by a high Q.E. $>35 \% @ 178 \mathrm{~nm}$ and a gain of a factor $5 \times 10^{6}$. The contamination measurements was performed with different techniques, such as high purity germanium screening, mass spectrometry 
and neutron activation. The total background from the different material contamination results to not limit the aimed sensitivity of the experiment [14]. The xenon is commercially obtained from the fractional distillation of the air, thus containing krypton contamination at a certain level. This is then a source of intrinsic background since a factor of $\sim 2 \times 10^{-11}$ of krypton occurs as the unstable isotope ${ }^{85} \mathrm{Kr}$, a $\beta$ emitter. The only way to reduce such krypton impurities is via distillation. So, before to start the physical run all the xenon will pass once in a krypton distillation column with the aim to reduce the levels of contamination to below the ppt level $\left(0.1 \times 10^{-12} \mathrm{Kr} / \mathrm{Xe}\right)$. The estimate of this intrinsic background results in about 0.15 events/year.

\subsection{Xenon storage}

XENON1T will handle xenon by means of a storage and recovery system named ReStoX. It has been installed in LNGS on August 2014 (see figure 4 (right)). It is a double-walled stainless steel sphere of $2.1 \mathrm{~m}$ diameter capable to store xenon in different thermal conditions, even in different phases, depending on the needs, under high purity conditions. For this purpose it is well insulated and equipped with sophisticated cooling systems able to provide cooling power with high response. The cooling system is based on (gaseous and liquid) nitrogen, so to make ReStoX functionalities totally independent from power failures. The stored xenon can be continuously purified thanks to a dedicated connection through a heat exchanger with the XENON1T purification system (which requires xenon in gaseous phase). ReStoX is kept at low pressure all the time (below a barg). In case of need of a recovery (voluntary or emergency-triggered) from the cryostat, the pressure in the cryostat (usually bigger than $2 \mathrm{barg}$ ) is enough to push liquid xenon through a dedicated recovery line in connection with ReStoX. Then ReStoX will be able to recover also most of the remaining gaseous xenon by subcooling the stored xenon and thanks to a direct gas-gas connection with the cryostat.

\section{XENON1T Upgrade}

Once completed, XENON1T will be the most sensitive worldwide dark matter experiment. It will reach its design sensitivity after two years of stable operations. A XENON1T upgrade is then foreseen, which will increase the amount of xenon to about 7 tons with an achievable goal of 20 ton-years of exposure. This will allow a sensitivity down to $3 \times 10^{-48} \mathrm{~cm}^{2}$ in the spin-independent WIMP-nucleon cross section. The construction of the detector upgrade is foreseen to start as early as 2018, and will run in parallel with the operation of XENON1T. Most of the detector installation have been conceived to be reused to host a larger volume TPC without modifications: only a new inner vessel of the cryostat, which will fit with the existing outer cryostat, and the TPC itself will be needed. Signal and high voltage cables for the new PMTs have already been installed within the cable pipes connecting the detector to the outside electronics and the existing DAQ, slow control, and computing infrastructure will be reused, as well as the cryogenic and purification systems for the operation of the new detector. However the main cost of the experiment will be procurement of additional xenon and PMTs. 


\section{Conclusion}

We have presented the results of XENON100, still running, and the XENON1T detector, installed in the Hall B of the LNGS, under construction. It is currently under commissioning and the beginning of data taking is foreseen at the end of 2015. With XENON1T and the planned upgrade, the XENON Collaboration will remain at the forefront of the exciting and challenging field of dark matter direct detection.

\section{References}

[1] W. Freeman and M. Turner, Measuring and understanding the universe, Rev. Mod. Phys. 751433 (2003)

[2] M.J. Jee et al., Discovery of a Ringlike Dark Matter Structure in the Core of the Galaxy Cluster Cl 0024+17, Astrophys. J. 661:728-749,2007 (2007)

[3] A. Bottino et al., Light neutralinos and WIMP direct searches, Phys. Rev. D 69037302 (2003)

[4] J. Ellis et al., Update on the direct detection of supersymmetric dark matter, Phys. Rev. D 71095007 (2005)

[5] A.Birkedal-Hansen and J. G. Wacker, Scalar dark matter from theory space, Phys. Rev. D 69065022 (2004)

[6] E. Aprile et al. (XENON100 Collaboration), The XENON100 dark matter experiment, Astropart. Phys. 35573 (2012)

[7] M.Yamashita et al., Scintillation response of liquid Xe surrounded by PTFE reflectors for gamma rays, NIM A $\mathbf{5 3 5}$ 692-698 (2004)

[8] E. Aprile et al. (XENON100 Collaboration), Material screening and selection for XENON100, Astropart. Phys. 3543 (2011)

[9] R. D. Peccei and H. R. Quinn, CP Conservation in the Presence of Instantons, Phys. Rev. Lett. 38, 1440 (1977)

[10] M. A. Shifman, A. I. Vainshtein and V. I. Zakharov, Can Confinement Ensure Natural CP Invariance of Strong Interactions?, Nucl. Phys. B 166, 493 (1980)

[11] L. F. Abbott and P. Sikivie, A Cosmological Bound on the Invisible Axion, Phys. Lett. B 120, 133 (1983)

[12] E. Aprile et al. [XENON100 Collaboration], First Axion Results from the XENON100 Experiment, Phys. Rev. D 90, no. 6, 062009 (2014)

[13] E. Aprile et al. [XENON1T Collaboration], Conceptual design and simulation of a water Cherenkov muon veto for the XENON1T experiment, JINST 9, 11006 (2014)

[14] E. Aprile et al. [XENON Collaboration], Lowering the radioactivity of the photomultiplier tubes for the XENON1T dark matter experiment, arXiv:1503.07698 [astro-ph.IM]. 